\title{
Conf.Researchr.Org: Towards a Domain-Specific Content Management System for Managing Large Conference Websites
}

\author{
Elmer van Chastelet Eelco Visser \\ Delft University of Technology, Netherlands \\ e.vanchastelet@tudelft.nl, visser@acm.org
}

\author{
Craig Anslow \\ Middlesex University, United Kingdom \\ c.anslow@mdx.ac.uk
}

\begin{abstract}
Federated conferences such as SPLASH are complex organizations composed of many parts (co-located conferences, symposia, and workshops), and are put together by many different people and committees. Developing the website for such a conference requires a considerable effort, and is often reinvented for each edition of a conference using software that provides little to no support for the domain. In this paper, we give a high-level overview of the design of Conf.Researchr.Org, a domain-specific content management system developed to support the production of large conference web sites, which is being used for the federated conferences of ACM SIGPLAN.
\end{abstract}

Categories and Subject Descriptors H.5.2 [Information interfaces and presentation (e.g., $\mathrm{HCI}$ )]: User Interfaces Graphical user interfaces

Keywords Conference Websites; Domain-Specific Content Management System

\section{Introduction}

Federated conferences such as SPLASH are complex organizations composed of many parts organized by volunteers. The main conference consists of multiple tracks and subconferences (OOPSLA, Onward!, SPLASH-I, invited speakers, tutorials, panels), and additional conferences, symposia, and workshops are co-located before the main conference. Each of the parts has its own steering, organizing, and program committees. For each of these parts, presentations (often based on papers) have to be selected. These presentations should be put together into a program that the attendees of the conference can inspect to decide what to attend. While

Permission to make digital or hard copies of part or all of this work for personal or classroom use is granted without fee provided that copies are not made or distributed for profit or commercial advantage and that copies bear this notice and the full citation on the first page. Copyrights for third-party components of this work must be honored. For all other uses, contact the Owner/Author.

Copyright is held by the owner/author(s).

SPLASH Companion'15, October 25-30, 2015, Pittsburgh, PA, USA ACM. 978-1-4503-3722-9/15/10

http://dx.doi.org/10.1145/2814189.2817270 there has been considerable attention for organizing and automating the paper submission and review process [1, 4], developing the website for such a conference also requires a considerable effort, and requires input from many people over an extended period of time. Often the process of developing it is reinvented for each yearly edition of a conference by a fresh team of volunteers using software that provides little to no support for the domain. As a result, the information for many tracks is not integrated in the main website. Each workshop maintains their own website from which the attendees have to harvest calls for papers and programs. In this paper, we give a high-level overview of the design of Conf.Researchr.Org, a domain-specific content management system developed to support the production of large conference web sites.

\section{Domain Model}

Figure 1 shows a domain model for the core of the application. While the model elides many aspects and details, it does show its main conceptual building blocks. The spine of the domain model (the yellow classes) represents events (things happening in time) at different levels of granularity. Each level represents a collection of the events at the next level. A ConferenceSeries represents the identity of a conference with regular (typically yearly) ConferenceEditions. A conference edition may have multiple co-located subconferences (including workshops and symposia), each of which are editions of their own series. For example, SLE is an independent conference series that often, but not always, co-locates with SPLASH. A sub-conference may have its own sub-conferences. For example, SLE organizes its own workshops. The main content of a conference edition is made up of Tracks. A track is a programmatic unit of a conference, that is, a series of Events (talks, keynotes, demonstrations, tutorials, panels) grouped in ProgramSessions. A track may have one (or more) CallForContributions and associated ImportantDates. The events making up a track typically correspond to the accepted papers for that track. The meta-data of accepted papers can be uploaded through an ImportRequest. We currently support the data format 


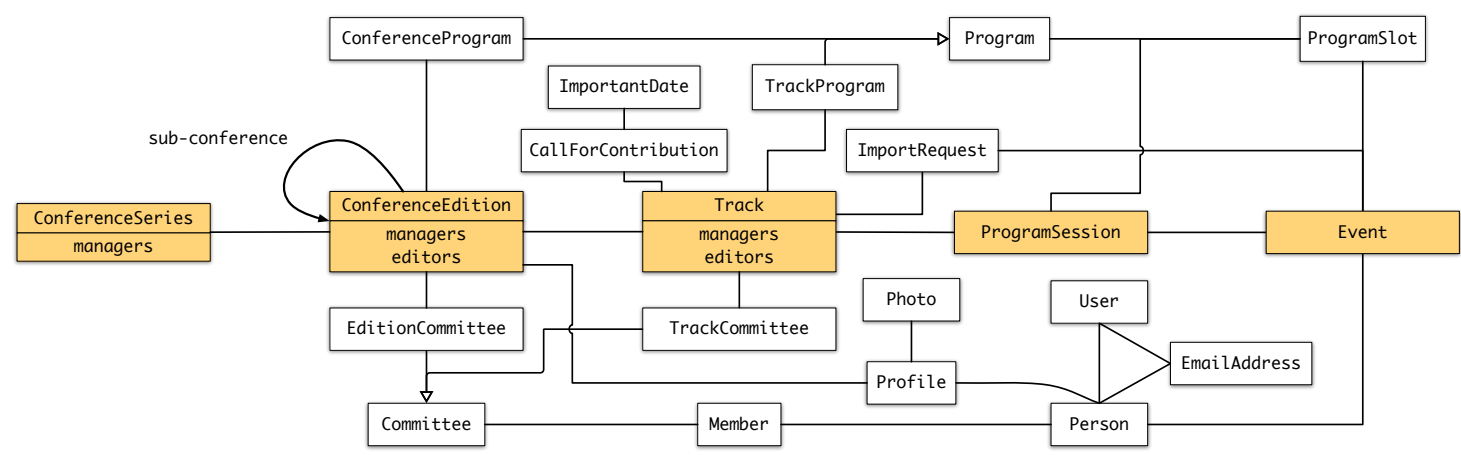

Figure 1. Domain model for the core of Conf.Researchr.Org.

exported by the HotCRP submission management system [4] as input to an ImportRequest.

The upper part of the domain model represents the ConferenceProgram, which integrates the TrackPrograms of all the tracks of the conference and its sub-conferences. Each track gets assigned ProgramSlots with a room and time allocation, which they can fill with ProgramSessions with Events. From these program data we generate a program page for the entire conference with faceted search to let attendees focus on specific tracks or days.

The lower part of the domain model concerns the people involved at various levels in the conference. A track typically has one or more TrackCommittees, such as an organizing committee, program committee, and external review committee, consisting of Members. For example, OOPSLA is a track of SPLASH with a separate PC and ERC. A conference edition typically has an organizing committee and a steering committee. Program sessions have chairs, and events have authors and/or speakers. Membership of a collection is linked to Persons, who are identified by an email address (which is not publicly displayed fon the site), and presented on the site with a profile consisting of a portrait, a bio, and their roles in the conference. When assigned some role, the person gets a notification email and is invited to activate their account in order to update their profile and the events they are involved with. For example, authors/speakers can attach media such as slides or videos to the page for their talk. Access to the upper levels is controled by means of a collection of managers who can administrate access control, and editors who can edit content.

\section{Implementation}

The conference management system is developed using WebDSL, a domain-specific programming language for the development of data rich web applications that integrates sub-languages for data modeling, a template language, access control rules, data validation, and full text search [6, 3, 5].

The system is implemented as a multi-tenant web application, hosting the web sites for multiple series of multiple conference editions in a single application. Multi-tenancy of the system is realized by using an identifier in the page URLs or the domain name that denotes the viewing context for each page. This viewing context is typically the conference itself which is being viewed, but may also be a parent conference in case of a co-located conference or workshop. The viewing context is used at various places in the implementation responsible for rendering the pages. It determines how navigation menus are constructed, whether or not a custom style sheet should be included and which, possibly roledependent, links a visitor has access to.

The application has been adopted by the main conferences in programming languages: SPLASH ('14,'15,'16), PLDI ('15,'16), ISMM'15, ESOP'15, ECOOP ('15,'16), POPL'16, PPoPP'16, Modularity'16, ICFP'16. There are many ideas for further improving the application such as improving the event scheduling workflow [2] and personalization of the conference schedule for attendees.

\section{Acknowledgments}

The initial development of the application was made possible by a contract with ACM SIGPLAN. We thank Jan Vitek for his support in realizing this contract and for being a critical early user. We thank the early adopters, especially Andrew Black for SPLASH 2014.

\section{References}

[1] Workshop on Organizing Workshops, Conferences, and Symposia for Computer Systems (WOWCS), 2008.

[2] L. Chilton, J. Kim, et al. Frenzy: collaborative data organization for creating conference sessions. In $C H I$, pages 1255-1264. ACM, 2014.

[3] D. M. Groenewegen and E. Visser. Declarative access control for WebDSL: Combining language integration and separation of concerns. In ICWE, pages 175-188, 2008.

[4] E. Kohler. Hot Crap! In WOWCS, 2008.

[5] E. van Chastelet. A domain-specific language for internal site search. Master's thesis, Delft University of Technology, 2013.

[6] E. Visser. WebDSL: A case study in domain-specific language engineering. In GTTSE, pages 291-373, 2007. 